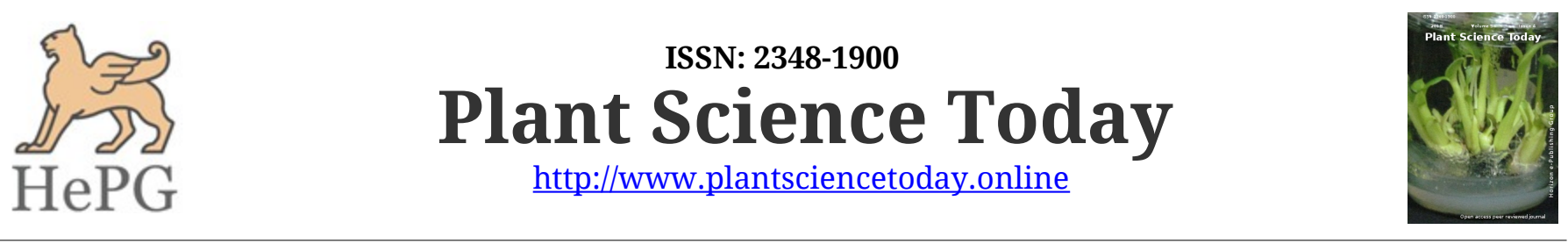

OPEN ACCESS

Review Article

\title{
Larvicidal activity of phytoextracts against dengue fever vector, Aedes aegypti - A review
}

\author{
K V Lakshmi ${ }^{1,2}$, A V Sudhikumar ${ }^{1}$, E M Aneesh ${ }^{2 *}$ \\ ${ }^{1}$ Department of Zoology, Christ College, Irinjalakuda, Kerala, India \\ ${ }^{2}$ Communicable Disease Research Laboratory, Department of Zoology, St. Joseph’s College, Irinjalakuda, Kerala, India
}

\section{Article history}

Received: 12 July 2018

Accepted: 20 September 2018

Published: 12 November 2018

\section{Editor}

Dr. V. Belavadi, University of Agricultural Sciences, Bangalore, India

\section{Publisher \\ Horizon e-Publishing Group}

\author{
*Correspondence \\ E. M. Aneesh \\ $凶$ aneeshembalil@gmail.com
}

\begin{abstract}
Since Aedes aegypti is considered as the major vector of dengue fever, development of strategies to accomplish improved vector control without much interference in the environment composition are more common. As phytochemicals are now in the run for achieving this goal, this review is a humble attempt to recognize the plant species and their larvicidal efficacy with their inhibitory action on the life cycle of the species of interest, that has been documented through various studies conducted till date. Here we also discuss the synergistic impact of a number of phytoextracts which will provide more efficient control measures for mosquito vectors. All these studies are an exploration for a risk-free vector control tactic to replace the current chemical insecticide application for the betterment of our nature.
\end{abstract}

\section{Keywords}

Aedes aegypti; phytoextracts; larvicidal activity; susceptibility

\section{Citation}

Lakshmi K V, Sudhikumar A V, Aneesh E M. Larvicidal activity of phytoextracts against dengue fever vector, Aedes aegypti - A review. Plant Science Today 2018;5(4):167-174. https://dx.doi.org/10.14719/pst.2018.5.4.407

\begin{abstract}
Copyright: (c) Lakshmi et al. (2018). This is an open-access article distributed under the terms of the Creative Commons Attribution License, which permits unrestricted use, distribution, and reproduction in any medium, provided the original author and source are credited (https://creativecommons.org/licenses/by/4.0/).
\end{abstract}

Indexing: Plant Science Today is covered by Scopus, CAS, AGRIS, CABI, Google Scholar, etc. Full list at http://www.plantsciencetoday.online

\section{Introduction}

Mosquitoes are considered as the principle vectors of several diseases affecting humans and animals which include dengue, filariasis, chickungunya, Japanese encephalitis, malaria, etc, which result in thousands of deaths every year. Among these, dengue fever is measured as one of the noxious diseases due to its high mortality rate and increasing pervasiveness (1). The rate of recurrence of dengue has grown significantly around the globe in current times. According to recent reports, it is estimated that almost three ninety million people across the world are infected per year of which ninety million are in severe state $(2,3)$. Most of the outbreaks of this disease remain unpredictable and are spreading to new areas, which result in an irrepressible increase in the occurrence of cases. Based on the data collected by National Vector Borne Disease Control Programme in India, dengue fever was first accounted in 1956 from Vellore 
District in Tamil Nadu and the first dengue haemorrhagic fever outburst was informed from the eastern coast in 1963 (4). Transmission of dengue fever virus to humans is through mosquitoes of the genus Aedes, mainly by Aedes aegypti, hence known as the most important dengue vector (5).

Research has proved that the most triumphant method for the diminution of such vector borne diseases is the successful control of their vector organisms. Different methods were pursued to implement this purpose from ancient periods including physical, chemical, mechanical, and biological methods, of which chemical method has been demonstrated to be the most effective.

Phytochemicals were used as a crucial mosquito vector managment tactic since 1920's and they were gradually replaced by the synthetic chemicals after the induction of DDT. In an earlier study, it was reported that the plant alkaloids resembling nicotine, anabasine, methyl anabasine and lupinine extracted from a weed Anabasis aphylla, had highly efficient larvicidal activity (6). Literature review in this sector suggested that more than 1200 plants were listed for their potential insecticidal value in the early 90's (7). They almost disappeared from the field after the discovery of the synthetic insecticides such as organochlorides, organophosphates, carbamates, DDT and pyrethroids (8).

Insecticides that contain compounds of high mortality rates have been used worldwide for the management of insect pests including mosquitoes. Even though these insecticides supported intrusions have efficiently controlled mosquito populations for several years, dependence on these limited active ingredients and their profound use has caused progression of resistance against these compounds (9). Studies on resistance development have suggested that, Aedes aegypti has triggered resistance to all insecticides including carbamates, organochlorides, organophosphates and pyrethroids (10). Resistance development and more prominently the environmental catastrophe have compelled the researchers to revert their corridors once again towards phytochemicals for achieving successful vector control devoid of such tribulations.

\section{Phytoextracts}

Botanicals are principally secondary metabolites that operate as a means of defence mechanism of the plants to resist continuous selection pressure from herbivore predators and other environmental factors and some of them exhibit natural insecticidal activity as well (11). An assessment of earlier studies gives comprehensive evidence for the utilization of plant products as insecticides against mosquito populations. Extraction and evaluation of phytochemicals from 150 plants were carried out and their larvicidal effects were listed by Hartzeall and Wilcoxon in 1941 (12). In a previous review performed on insecticides derived from plants, covering a period of 1941 to 1953, it was reported that several phytochemicals were in use against mosquitoes for their control (13). Developing resistance against chemical insecticides made this harmless method more popular amongst researchers and common people alike. Present studies are focusing on these phytoextract based vector control strategies and also developing a newer and more effective method called synergy where a combination of more than one compound either of plant products or a combination of plant product and chemical insecticides are used for accomplishing the objective.

Same phytochemicals exhibit different susceptibility status to different mosquito species. Among the key vector genera like Aedes and Culex, Aedes mosquitoes are less susceptible to insecticides and botanicals (11). Since resistance development and environmental peril have been revealed for conventional insecticides, a range of studies on plant products as the mosquito vector control measures are being carried out by the researchers. Wide array of these natural compounds have been tested for this purpose including thousands of plant species. In this review, the plant extracts that have accounted for larvicidal efficacy against Aedes mosquitoes and their lethal dose are listed (Table 1). Various plants having larvicidal efficacy were subjected to further studies for identification of the chemical compounds which specifically possess larvicidal activity. Many compounds of interest have been recognized and isolated from various plant species of which important ones are listed in Table 2.

To compete with the increasing resistance development in mosquito species, a newer and faster method of vector control has been formulated in which synergistic action of more than one insecticide is exploited in combination with one another to enhance their potency for mosquito control. Studies have proven that the synergistic combination of biological and chemical insecticides yield a promising alternative resolution in vector management (14). At present, many synergistic combinations are under experimentation which includes the synergy of chemical and biological insecticides and mixtures of different phytoextracts alone.

When the leaf extracts of Solidago canadensis and Eugenia jambolana were combined with deltamethrin for the synergistic activity had a synergistic factor of 4.09 and 1.80 . They were observed to give more efficient control than the phytoextracts alone (15). Synergistic efficacy of Vitex negundo, Clerodendrum inerme and Gliricidia sepium with Pongamia glabra against Aedes aegypti provided a synergistic factor of 1.90, 1.50 and 1.72 respectively and revealed that synergy of phytoextracts also offer proficient vector control in which only the combinations of phytoextracts were entailed (16). 
Table 1: Efficacy of botanical extracts in controlling/reducing the populations of Aedes aegypti, a dengue fever vector (LC: Lethal Concentration, ppm: parts per million, mg/L: milligram per liter)

\begin{tabular}{|c|c|c|c|c|c|}
\hline Plant species & Plant families & Plant part & Solvent & $\begin{array}{l}\text { Lethal concentrations } \\
\text { or biological activity }\end{array}$ & References \\
\hline Caulerpa scalpelliformis & Caulerpaceae & Whole & Acetone & LC: $53.7 \mathrm{mg} / \mathrm{L}$ & (21) \\
\hline Cannabis sativa & Cannabaceae & Leaf & Ethanol & LC: $5000 \mathrm{mg} / \mathrm{L}$ & (22) \\
\hline Codiaeum variegatum & Euphorbiaceae & Leaf & Water & LC: $37,600 \mathrm{mg} / \mathrm{L}$ & (23) \\
\hline Azadirachta indica & Meliaceae & Leaf & Water & LC: $4800 \mathrm{mg} / \mathrm{L}$ & (23) \\
\hline Annona squamosa & Annonaceae & Leaf & Water & LC: $2400 \mathrm{mg} / \mathrm{L}$ & (23) \\
\hline Azadirachita indica & Meliaceae & Seed & Crushed seeds & LC: 100 (59) mg/L & (24) \\
\hline Angelico glauca & Apiaceae & Seeds & Commercial oils & LC: $52-74 \mathrm{mg} / \mathrm{L}$ & (25) \\
\hline Calophyllum inophyllum & Calophyllaceae & Leaf & Ethyl-acetate fraction & LC: $35.49 \mathrm{mg} / \mathrm{L}$ & (26) \\
\hline Calophyllum inophyllum & Calophyllaceae & Seed & Ethyl-acetate fraction & LC: $8.2 \mathrm{mg} / \mathrm{L}$ & (26) \\
\hline Alnus glutinosa & Betulaceae & Old litter & Polyphenols & LC: $200-400 \mathrm{mg} / \mathrm{L}$ & (17) \\
\hline Abuta grandifolia & Menispermaceae & Fruit & Dichloro methane & LC: $2.6 \mathrm{mg} / \mathrm{L}$ & (27) \\
\hline Feronia limonia & Rutaceae & Leaf & Acetone & $\begin{array}{l}\mathrm{LC}_{50}: 57.23 \\
\mathrm{LC}_{90}: 146.21 \mathrm{mg} / \mathrm{L}\end{array}$ & (28) \\
\hline Alnus glutinosa & Betulaceae & $\begin{array}{l}\text { Different } \\
\text { aged litter }\end{array}$ & & $\mathrm{LC}_{50}: 0.382 \mathrm{~g} / \mathrm{L}$ & (18) \\
\hline Cassia obtusifolia & Fabaceae & Seed & Methanol & LC: 40 (51) mg/L & (29) \\
\hline$\overline{\text { Cassia tora }}$ & Caesalpinaceae & Seed & Methanol & LC:20 (59) mg/L & (29) \\
\hline Callitris glaucophylla & Cupressaceae & Wood & Steam distilled & LC:0.69 mg/L & (30) \\
\hline Cassia obtusifolia & Leguminosae & Seed & Chloroform & $\mathrm{LC}_{50:} 1.4 \mathrm{ppm}$ & (31) \\
\hline Cassia tora & Caesulpinaceae & Seed & Methanol & $\mathrm{LC}_{50}: 20 \mathrm{mg} / \mathrm{l}$ & (31) \\
\hline Piper retrofractum & Piparaceae & $\begin{array}{l}\text { Unripe and } \\
\text { ripe fruit }\end{array}$ & & $\mathrm{LC}_{50}: 79 \mathrm{ppm}$ & (32) \\
\hline Curcuma aromatic & Zingiberaceae & Rhizome & Hexane & $\mathrm{LC}_{50}: 36.30 \mathrm{ppm}$ & (33) \\
\hline Rhinacanthus nasutus & Acanthaceae & Root & Petroleum ether & $\begin{array}{l}\mathrm{LC}_{50}: 3.93 \mathrm{ppm} \\
\mathrm{LC}_{90}: 18.51 \mathrm{ppm}\end{array}$ & (34) \\
\hline Derris elliptica & Fabaceae & Root & Petroleum ether & $\begin{array}{l}\mathrm{LC}_{50}: 11.17 \mathrm{ppm} \\
\mathrm{LC}_{90}: 32.22 \mathrm{ppm}\end{array}$ & (34) \\
\hline Homalomena aromatica & Araceae & Whole plant & Petroleum ether & $\begin{array}{l}\mathrm{LC}_{50}: 40.36 \mathrm{ppm} \\
\mathrm{LC}_{90}: 77.21 \mathrm{ppm}\end{array}$ & (34) \\
\hline Momordica charantia & Cucurbitaceae & Fruit & Hexane & $\begin{array}{l}\mathrm{LC}_{50}: 122.45 \mathrm{ppm} \\
\mathrm{LC}_{90}: 191.86 \mathrm{ppm}\end{array}$ & (35) \\
\hline Ocimum basilium & Lamiaceae & Leaf & Water & $\begin{array}{l}\mathrm{LC}_{50}: .4 .66 \mathrm{mg} / \mathrm{ml} \\
\mathrm{LC}_{90}: 8.74 \mathrm{mg} / \mathrm{ml}\end{array}$ & (36) \\
\hline Albizzia amara & Fabaceae & Leaf & Water & $\begin{array}{l}\mathrm{LC}_{50}: 7.10 \mathrm{mg} / \mathrm{mi} \\
\mathrm{LC}_{90}: 13.23 \mathrm{mg} / \mathrm{ml}\end{array}$ & (36) \\
\hline Ageratina adenophora & Asteraceae & Twigs & Acetone & $\mathrm{LC}_{50}: 356.70 \mathrm{ppm}$ & (37) \\
\hline Millingntonia hortensis & Bignoniaceace & Leaf & Aectone & $\mathrm{LC}_{50}: 104.70 \mathrm{ppm}$ & (38) \\
\hline Ocimum sanctum & Labiate & Leaf & Acetone & $\mathrm{LC}_{50}: 425.94 \mathrm{ppm}$ & (38) \\
\hline Citrullus colocynthis & Cucurbitaceae & Leaf & Petroleum ether & $\begin{array}{l}\mathrm{LC}_{50}: 74.57 \mathrm{ppm} \\
\mathrm{LC}_{90}: 538.30 \mathrm{ppm}\end{array}$ & (39) \\
\hline Coccinia indica & Cucurbitaceae & Leaf & Methanol & $\begin{array}{l}\mathrm{LC}_{50}: 309.46 \mathrm{ppm} \\
\mathrm{LC}_{90}: 1330.43 \mathrm{ppm}\end{array}$ & (39) \\
\hline Cucumis sativus & Cucurbitaceae & Leaf & Methanol & $\begin{array}{l}\mathrm{LC}_{50}: 492.73 \mathrm{ppm} \\
\mathrm{LC}_{90}: 1824.20 \mathrm{ppm}\end{array}$ & (39) \\
\hline Momordica charantia & Cucurbitaceae & Leaf & Methanol & $\begin{array}{l}\mathrm{LC}_{50}: 199.14 \mathrm{ppm} \\
\mathrm{LC}_{90}: 780.10 \mathrm{ppm}\end{array}$ & (39) \\
\hline Trichosanthes anguina & Cucurbitaceae & Leaf & Acetone & $\begin{array}{l}\mathrm{LC}_{50}: 554.20 \mathrm{ppm} \\
\mathrm{LC}_{90}: 2235.34 \mathrm{ppm}\end{array}$ & (39) \\
\hline Solanum nigrum & Solanaceae & Dried fruit & Hexane & $\begin{array}{l}\mathrm{LC}_{50}: 17.63 \mathrm{ppm} \\
\mathrm{LC}_{90}: 65.22 \mathrm{ppm}\end{array}$ & (15) \\
\hline Fius bengahalensis & Moraceae & Leaf & Methanol & $\begin{array}{l}\mathrm{LC}_{50}: 70.29 \mathrm{ppm} \\
\mathrm{LC}_{90}: 137.23 \mathrm{ppm}\end{array}$ & $(40)$ \\
\hline Euodia rideleyi & Rutaceae & Leaf & Ethyl acetate & $\begin{array}{l}\mathrm{LC}_{50}: 139.80 \mathrm{ppm} \\
\mathrm{LC}_{90}: 203.50 \mathrm{ppm}\end{array}$ & $(41)$ \\
\hline
\end{tabular}


Table 1: Contd.

\begin{tabular}{|c|c|c|c|c|c|}
\hline Plant species & Plant families & Plant part & Solvent & $\begin{array}{l}\text { Lethal concentrations } \\
\text { or biological activity }\end{array}$ & References \\
\hline Enteromorpha intestinalis & Ulvaceae & Whole plant & $\begin{array}{l}\text { Dimethyl sulfoxide } \\
\text { (DMSO) }\end{array}$ & $\begin{array}{l}\mathrm{LC}_{50}: 0.0744 \mathrm{mg} / \mathrm{ml} \\
\mathrm{LC}_{90}: 0.1399 \mathrm{mg} / \mathrm{ml}\end{array}$ & $(42)$ \\
\hline Dictyota dichotoma & Dictyotaceae & Whole plant & $\begin{array}{l}\text { Dimethyl sulfoxide } \\
\text { (DMSO) }\end{array}$ & $\begin{array}{l}\mathrm{LC}_{50}: 0.0683 \mathrm{ppm} \\
\mathrm{LC}_{90}: 0.1401 \mathrm{ppm}\end{array}$ & $(42)$ \\
\hline Ervatamia coronaria & Apocynaceae & Leaf & Benzene & $\begin{array}{l}\mathrm{LC}_{50}: 89.59 \mathrm{ppm} \\
\mathrm{LC}_{90}: 166.04 \mathrm{ppm}\end{array}$ & $(43)$ \\
\hline Caesalpinia pulcherrima & Fabaceae & Leaf & Benzene & $\begin{array}{l}\mathrm{LC}_{50}: 136.36 \mathrm{ppm} \\
\mathrm{LC}_{90}: 272.15 \mathrm{ppm}\end{array}$ & $(43)$ \\
\hline Mentha piperita & Lamiaceae & Essential oil & Ethanol & $\begin{array}{l}\mathrm{LC}_{50}: 111.9 \mathrm{ppm} \\
\mathrm{LC}_{90}: 295.18 \mathrm{ppm}\end{array}$ & (44) \\
\hline Morinda citrifolia & Rubiaceae & Leaf & methanol & $\begin{array}{l}\mathrm{LC}_{50}: 277.92 \mathrm{ppm} \\
\mathrm{LC}_{90}: 568.18 \mathrm{ppm}\end{array}$ & $(45)$ \\
\hline Acalypha alnifolia & Euphorbiaceae & Leaf & methanol & $\begin{array}{l}\mathrm{LC}_{50}: 128.55 \mathrm{ppm} \\
\mathrm{LC}_{90}: 381.67 \mathrm{ppm}\end{array}$ & $(46)$ \\
\hline Calotropis gigantean & Asclepiadaceae & Leaf & Ethanol & $\begin{array}{l}\mathrm{LC}_{50}: 136.48 \mathrm{ppm} \\
\mathrm{LC}_{90}: 327.72 \mathrm{ppm}\end{array}$ & $(47)$ \\
\hline Citrus sinensis & Rutaceae & Fruit & Ethanol & $\begin{array}{l}\mathrm{LC}_{50}: 342.45 \mathrm{ppm} \\
\mathrm{LC}_{90}: 734.98 \mathrm{ppm}\end{array}$ & $(48)$ \\
\hline Aloe vera & Liliaceae & Leaf & Petroleum ether & $\begin{array}{l}\mathrm{LC}_{50}: 253.30 \mathrm{ppm} \\
\mathrm{LC}_{90}: 563.18 \mathrm{ppm}\end{array}$ & (49) \\
\hline Sphaeranthus indicus & Asteraceae & Whole plant & Ethyl acetate & $\begin{array}{l}\mathrm{LC}_{50}: 201.11 \mathrm{ppm} \\
\mathrm{LC}_{90}: 865.83 \mathrm{ppm}\end{array}$ & $(50)$ \\
\hline Citrullus colocynthis & Cucurbitaceae & Whole plant & Dichloromethane & $\begin{array}{l}\mathrm{LC}_{50}: 515.69 \mathrm{ppm} \\
\mathrm{LC}_{90}: 1725.59 \mathrm{ppm}\end{array}$ & $(50)$ \\
\hline Abutilon indicum & Malvaceae & Leaf & Hexane & $\begin{array}{l}\mathrm{LC}_{50}: 261.31 \mathrm{ppm} \\
\mathrm{LC}_{90}: 1196.20 \mathrm{ppm}\end{array}$ & $(50)$ \\
\hline Cleistanthus collinus & Euphorbiaceae & Leaf & Ethyl acetate & $\begin{array}{l}\mathrm{LC}_{50}: 560.41 \mathrm{ppm} \\
\mathrm{LC}_{90}: 2669.86 \mathrm{ppm}\end{array}$ & $(50)$ \\
\hline Leucas aspera & Lamiaceae & Leaf & Ethyl acetate & $\begin{array}{l}\mathrm{LC}_{50}: 483.21 \mathrm{ppm} \\
\mathrm{LC}_{90}: 3195.91 \mathrm{ppm}\end{array}$ & $(50)$ \\
\hline Murraya koenigii & Rutaceae & Leaf & Diethyl ether & $\begin{array}{l}\mathrm{LC}_{50}: 511.12 \mathrm{ppm} \\
\mathrm{LC}_{90}: 1882.24 \mathrm{ppm}\end{array}$ & $(50)$ \\
\hline Hyptis suaveolens & Lamiaceae & Aerial parts & Hexane & $\begin{array}{l}\mathrm{LC}_{50}: 543.66 \mathrm{ppm} \\
\mathrm{LC}_{90}: 3546.69 \mathrm{ppm}\end{array}$ & $(50)$ \\
\hline Terminalia chebula & Combretaceae & Leaves & Methanol & $\begin{array}{l}\mathrm{LC}_{50: 93.24} \mathrm{ppm} \\
\mathrm{LC}_{90}: 186.76 \mathrm{ppm}\end{array}$ & (51) \\
\hline Senecio laetus & Asteraceae & Root & Methanol & $\begin{array}{l}\mathrm{LC}_{50:} 22.30 \mathrm{ppm} \\
\mathrm{LC}_{90}: 144.67 \mathrm{ppm}\end{array}$ & (52) \\
\hline
\end{tabular}

\section{Mode of action of phytoextracts}

A review on mode of action of phytoextracts revealed that only a few studies have been carried out in this area and there is a lot more to survey (17). However, the studies performed suggest that botanical derivatives have a major effect on the mid gut epithelium and a lesser effect on the gastric area and malpighian tubules (18). Most secondary metabolites of the plants are responsible for the toxic effect which results in insecticidal activity having a wide array of nonspecific molecular targets including proteins (enzymes, receptors, signaling molecules, ionchannels and structural proteins), nucleic acids, bio-membranes, and other cellular components (19). Mode and site of action of some of the secondary metabolites of plants have been identified and are listed in this review (Table 3).

\section{Effects of phytoextracts on Aedes aegypti other than larvicidal activity}

Apart from larvicidal efficacy, some phytoextracts exhibit characteristics which are involved in the inhibition of mosquito growth and reproductive capacity. Some of the foremost physiological characteristics like developmental period, growth, adult emergence, fecundity, fertility and egg hatching are affected by these factors, and hence they are prevalently known as Insect Growth Regulators (IGRs), which can be incorporated in mosquito vector control. Over one thousand plant species contain such compounds including phytoecdysones, anti-juvenile hormones and 
Table 2: Common active compounds of interest isolated from plant species having larvicidal efficacy

\begin{tabular}{|c|c|c|c|}
\hline Active Compounds of Interest & Structure & Plant Species & Reference \\
\hline (5E)-ocimenone & & Tagetes minuta & (53) \\
\hline Azadirachtin & & Azadirachta indica & (54) \\
\hline Rotenone & & Derris elliptica & (55) \\
\hline Capillin & & Artemisia nilagirica & (56) \\
\hline Quassin & & Quassia amara & (57) \\
\hline Neolignans & & Piper decurrens & (58) \\
\hline Arborine & & Glycosmis pentaphylla & (59) \\
\hline
\end{tabular}

Table 3: Mode and site of action of some of the secondary metabolites of plants having larvicidal efficacy

\begin{tabular}{lll}
\hline Secondary metabolites of plants & Mode and site of action & Reference \\
\hline Essential oils & Inhibition of acetylecholinestrase & \\
\hline Thymol & Inhibition of GABA-gated chloride channel \\
\hline Pyrethrin & Sodium and potassium ion exchange disruption \\
\hline Rotenone & Inhibition of cellular respiration & (19) \\
\hline Ryanodine & Blockage of calcium channels \\
\hline Azadirachtin & Hormonal balance disruption, mitotic poisoning \\
\hline
\end{tabular}

phyto-juvenoids, which act as IGRs (20). Some of the important botanical extracts comprising growth and development regulating aptitude are listed in Table 4.

\section{Conclusion}

A wide variety of flora around the world has been screened against the major dengue fever vector, Aedes aegypti, and identified with their ability to control this vector species either by their insecticidal capability or by other means like growth and development inhibition. These phytoextracts have established their potential and could be constructive in the partial or complete replacement of the chemical insecticides, which presently is the major means of vector control. This will be beneficial, ruling out environmental hazards including harmful effects on non-target organisms. One of the chief challenges that could 
Table 4: Effect of selected phytoextracts on growh, development, reproduction, abnormalities, hatch rates and fertility of Aedes aegypti

\begin{tabular}{|c|c|c|c|c|}
\hline Plant species & Solvent/Fraction/compound & $\mathrm{Mg} / \mathrm{L}$ & Effect on Aedes aegypti & Reference \\
\hline \multirow{2}{*}{ Azadirachta indica } & Water & 100 & $\begin{array}{l}\text { 80.5\% pupal - adult intermediates } \\
\text { mortality and } 3 \% \text { pupal - adult }\end{array}$ & \multirow{2}{*}{$(60)$} \\
\hline & Methyl-tert.-butylether/water & 20 & $\begin{array}{l}\text { intermediates exhibited protruding } \\
\text { wing-sheath and mouthparts }\end{array}$ & \\
\hline Albizzia lebbeck & Ethyl alcohol & 500 & $95 \%$ reduced adult emergence at day- 7 & $(61)$ \\
\hline $\begin{array}{l}\text { Calophyllum } \\
\text { inophyllum }\end{array}$ & Ethyl acetate fraction (seeds) & 0.22 & $\mathrm{EC}_{50}$ for inhibition of adult emergence & (26) \\
\hline Cassia holosericea & Ethyl alcohol & 1000 & Prevented pupation up to day-7 & $(62)$ \\
\hline Cyperus iria & Leaf aged $1 \& 2$ month & 1000 & $100 \%$ reduced emergence & (63) \\
\hline $\begin{array}{l}\text { Ervatamia } \\
\text { coronaria }\end{array}$ & Petroleum ether & 1000 & Prevented pupation up to day-7 & $(61)$ \\
\hline Melia volkensii & $\begin{array}{l}\text { Hexane/Ethyl acetate } \\
\text { fraction (1:1) }\end{array}$ & 20 & $\begin{array}{l}100 \% \text { mortality during molting and } \\
100 \% \text { reduced emergence }\end{array}$ & (63) \\
\hline Azadirachta indica & Oils from crushed seeds & 50 & $\begin{array}{l}\text { Treated larvae developed to pupae but } \\
\text { failed to emerge }\end{array}$ & (24) \\
\hline $\begin{array}{l}\text { Oligochaeta } \\
\text { ramose }\end{array}$ & Acetone & 50 & $\begin{array}{l}74 \% \text { larval mortality (larval -pupal } \\
\text { intermediate) and } 76 \% \text { reduced } \\
\text { emergence }\end{array}$ & $(64)$ \\
\hline Rhazya stricta & Partially purified asewerine & 100 & $86 \%$ emergence at day-7 & $(65)$ \\
\hline $\begin{array}{l}\text { Rhinacanthus } \\
\text { nasutus }\end{array}$ & Petroleum ether fraction & 2.9 & $\mathrm{EC}_{50}$ for inhibition of adult emergence & $(26)$ \\
\hline Solanum suratense, & Ethyl acetate fraction (leaf) & $\mathrm{EC} 50 / 2$ & $40 \%$ decrease in egg hatching & (65) \\
\hline
\end{tabular}

get eluded is resistance development in the particular vector species.

This review is a preface to the manipulation of vector management strategies using phytoextracts, which is now in its budding stage, but should come to the public interest so that we can conserve our nature without spreading diseases.

\section{Acknowledgements}

The authors are thankful to the Principal, St. Joseph's College, Irinjalakuda for the laboratory facilities provided. We acknowledge UGC Major Research Project (F. No. 42/609/2013 (SR)) and KSCSTE (F. No. 027/SRSLS/2013/CSTE) for providing funds.

\section{Conflict of Interest}

The authors declare no conflict of interest.

\section{Authors' contribution}

KVL has done the collection of literature and prepared the content of the manuscript, AVS and EMA provided inputs and supervised the work.

\section{References}

1. World Health Organization, Special Programme for Research, Training in Tropical Diseases, World Health Organization. Department of Control of Neglected Tropical Diseases, World Health Organization. Epidemic, Pandemic Alert. Dengue: guidelines for diagnosis, treatment, prevention and control. World Health Organization; 2009.

2. Bhatt S, Gething PW, Brady OJ, Messina JP, Farlow AW, Moyes CL, Drake JM, Brownstein JS, Hoen AG, Sankoh O, Myers MF. The global distribution and burden of dengue. Nature 2013;496(7446):504. https://doi.org/10.1038/nature12060

3. Brady OJ, Gething PW, Bhatt S, Messina JP, Brownstein JS, Hoen AG, Moyes CL, Farlow AW, Scott TW, Hay SI. Refining the global spatial limits of dengue virus transmission by evidence-based consensus. PLoS neglected tropical diseases. 2012 ;6(8):e1760. https://doi.org/10.1371/journal.pntd.0001760

4. National Vector Borne disease control programme, Guidelines for Management of Dengue Fever, Dengue Haemorrhagic Fever and Dengue Shock Syndrome. 2008.

5. Simmons CP, Farrar JJ, van Vinh Chau N, Wills B. Dengue. New England Journal of Medicine. 2012;366(15):1423-32. https://doi.org/10.1056/NEJMra1110265

6. Campbell FL, Sullivan WN, Smith CR. The relative toxicity of nicotine, anabasine, methyl anabasine, and lupinine for culicine mosquito larvae. Journal of Economic entomology. $1933 \quad$ Apr 1;26(2):500-9. https://doi.org/10.1093/jee/26.2.500

7. McIndoo NE. Plants of possible insecticidal value: a review of the literature up to 1941. US Department of Agriculture, Agricultural Research Administration, 
Bureau of Entomology and Plant Quarantine; 1945. https://doi.org/10.5962/bhl.title.122356

8. Ghosh A, Chowdhury N, Chandra G. Plant extracts as potential mosquito larvicides. The Indian journal of medical research. 2012 May;135(5):581.

9. Brown AW. Insecticide resistance in mosquitoes: a pragmatic review. Journal of the American Mosquito Control Association. 1986 Jun;2(2):123-40.

10. Ranson H, Burhani J, Lumjuan N, Black IV WC. Insecticide resistance in dengue vectors. TropIKA. net [online]. 2010;1(1).

11. Shaalan EA, Canyon D, Younes MW, Abdel-Wahab H, Mansour AH. A review of botanical phytochemicals with mosquitocidal potential. Environment international. 2005;31(8):1149-66. https://doi.org/10.1016/ j.envint.2005.03.003

12. Hartzell A, Wilcoxon F. A survey of plant products for insecticidal properties. Contrib Boyce Thompson Inst. 1941; 12:127-41.

13. Jacobson M. Insecticides from plants: a review of the literature, 1941-1953. United States Department of Agriculture; Washington; 1958.

14. Rodrigues AM, De Paula JE, Roblot F, Fournet A, Espíndola LS. Larvicidal activity of Cybistax antisyphilitica against Aedes aegypti larvae. Fitoterapia. 2005;76(7-8):755-7. https://doi.org/10.1016/j.fitote.2005.08.015

15. Raghavendra K, Singh SP, Subbarao SK, Dash AP. Laboratory studies on mosquito larvicidal efficacy of aqueous \& hexane extracts of dried fruit of Solanum nigrum Linn. Indian Journal of Medical Research. $2009 \mathrm{Jul}$ 1;130(1):74

16. Yankanchi S, Yadav OV, Jadhav GS. Synergistic and individual efficacy of certain plant extracts against dengue vector mosquito, Aedes aegypti. J. Biopest. 2014 Jan $1 ; 7: 22-8$.

17. Rey D, Cuany A, Pautou MP, Meyran JC. Differential sensitivity of mosquito taxa to vegetable tannins. Journal of Chemical Ecology. 1999 Mar 1;25(3):537-48. https://doi.org/10.1023/A:1020953804114

18. David JP, Rey D, Pautou MP, Meyran JC. Differential toxicity of leaf litter to dipteran larvae of mosquito developmental sites. Journal of invertebrate pathology. 2000;75(1):9-18. https://doi.org/10.1006/jipa.1999.4886

19. Rattan RS. Mechanism of action of insecticidal secondary metabolites of plant origin. Crop protection. 2010 Sep 1;29(9):913-20. https://doi.org/10.1016/j.cropro.2010.05.008

20. Varma J, Dubey NK. Prospectives of botanical and microbial products as pesticides of tomorrow. Current science. 1999 Jan 25:172-9.

21. Thangam TS, Kathiresan K. Mosquito larvicidal activity of marine plant extracts with synthetic insecticides. Botanica Marina. 1991;34(6):537-40. https://doi.org/10.1515/botm.1991.34.6.537

22. Jalees S, Sharma SK, Rahman SJ, Verghese T. Evaluation of insecticidal properties of an indigenous plant, Cannabis sativa Linn., against mosquito larvae under laboratory conditions. Journal of Entomological Research. 1993;17(2):117-20.

23. Monzon RB, Alvior JP, Luczon LL, Morales AS, Mutuc FE. Larvicidal potential of five Philippine plants against Aedes aegypti (Linnaeus) and Culex quinquefasciatus (Say). The Southeast Asian journal of tropical medicine and public health. 1994 Dec;25(4):755-9.

24. Sinniah B, Sinniah D, Ibrahim J. Effect of neem oil and mosquito larvae. Mosq Borne Dis Bull. 1994; 1:90-3.
25. Sharma RN, Deshpande SG, Tungikar VB, Joseph M. Toxicity of natural essential oils to mosquitoes, Aedes aegypti and Culex fatigans. Geobios. 1994; 21:162-5.

26. Pushpalatha E, Muthukrishnan J. Efficacy of two tropical plant extracts for the control of mosquitoes. Journal of applied entomology. 1999 Jul;123(6):369-73. https://doi.org/10.1046/j.1439-0418.1999.00373.x

27. Ciccia G, Coussio J, Mongelli E. Insecticidal activity against Aedes aegypti larvae of some medicinal South American plants. Journal of Ethnopharmacology. 2000 Sep 1;72(12):185-9. https://doi.org/10.1016/S0378-8741(00)00241-5

28. Rahuman AA, Gopalakrishnan G, Ghouse BS, Arumugam S, Himalayan B. Effect of Feronia limonia on mosquito larvae. Fitoterapia. $\quad 2000 \quad$ Sep 1;71(5):553-5. https://doi.org/10.1016/S0367-326X(00)00164-7

29. Jang YS, Baek BR, Yang YC, Kim MK, Lee HS. Larvicidal activity of leguminous seeds and grains against Aedes aegypti and Culex pipiens pallens. Journal of the American Mosquito Control Association. 2002 Sep;18(3):210-3.

30. Shaalan E, Canyon DV, Faried MW, Abdel-Wahab H, Mansour A. Efficacy of a highly active phytochemical (Callitris glaucophylla) against mosquito vectors of dengue and Japanese encephalitis. In: The annual Queensland Health and Medical Scientific Meeting, "Making it better: Encouraging health research and innovation. 2003 Nov 2526 Brisbane (pp. 25-26).

31. Yang YC, Lim MY, Lee HS. Emodin isolated from Cassia obtusifolia (Leguminosae) seed shows larvicidal activity against three mosquito species. Journal of agricultural and food chemistry. 2003 Dec 17;51(26):7629-31. https://doi.org/10.1021/jf034727t

32. Chansang U, Zahiri NS, Bansiddhi J, Boonruad T, Thongsrirak P, Mingmuang J, Benjapong N, Mulla MS. Mosquito larvicidal activity of aqueous extracts of long pepper (Piper retrofractum Vahl) from Thailand. Journal of Vector Ecology. 2005 Dec 1;30(2):195.

33. Choochote W, Chaiyasit D, Kanjanapothi D, Rattanachanpichai E, Jitpakdi A, Tuetun B, Pitasawat B. Chemical composition and anti-mosquito potential of rhizome extract and volatile oil derived from Curcuma aromatica against Aedes aegypti (Diptera: Culicidae). Journal of vector ecology. 2005 Dec 1;30(2):302.

34. Komalamisra N, Trongtokit Y, Rongsriyam Y, Apiwathnasorn C. Screening for larvicidal activity in some Thai plants against four mosquito vector species. Southeast Asian Journal of Tropical Medicine and Public Health. 2005 Nov 1;36(6):1412.

35. Singh RK, Dhiman RC, Mittal PK. Mosquito larvicidal properties of Momordica charantia Linn (family: Cucurbitaceae). Journal of Vector Borne Diseases. 2006 Jun;43(2):88.

36. Murugan K, Murugan P, Noortheen A. Larvicidal and repellent potential of Albizzia amara Boivin and Ocimum basilicum Linn against dengue vector, Aedes aegypti (Insecta: Diptera: Culicidae). Bioresource Technology. 2007 Jan 1;98(1):198-201. https://doi.org/10.1016/j.biortech.2005.12.009

37. Mohan DR, Ramaswamy M. Evaluation of larvicidal activity of the leaf extract of a weed plant, Ageratina adenophora, against two important species of mosquitoes, Aedes aegypti and Culex quinquefasciatus. African Journal of Biotechnology. 2007 Mar 5;6(5):631-8.

38. Kaushik R, Saini P. Larvicidal activity of leaf extract of Millingtonia hortensis (Family: Bignoniaceae) against Anopheles stephensi, Culex quinquefasciatus and Aedes aegypti. Journal of vector borne diseases. 2008 Mar 1;45(1):66.

39. Rahuman AA, Venkatesan P. Larvicidal efficacy of five cucurbitaceous plant leaf extracts against mosquito 
species. Parasitology research. 2008;103(1):133. https://doi.org/10.1007/s00436-008-0940-5

40. Govindarajan M. Larvicidal efficacy of Ficus benghalensis L. plant leaf extracts against Culex quinquefasciatus Say. European review for medical and pharmacological sciences. 2010; 14:107-11.

41. Prathibha KP, Raghavendra BS, Vijayan VA. Evaluation of larvicidal effect of Euodia ridleyi Hochr. Leaf extract against three mosquito species at Mysore. Research Journal of Biological Sciences. 2010;5(6):452-5. https://doi.org/10.3923/rjbsci.2010.452.455

42. Beula JM, Ravikumar S, Ali MS. Mosquito larvicidal efficacy of seaweed extracts against dengue vector of Aedes aegypti. Asian Pacific Journal of Tropical Biomedicine. $2011 \quad$ Oct $1 ; 1(2): \quad$ S143-6. https://doi.org/10.1016/S2221-1691(11)60143-3

43. Govindarajan M, Mathivanan T, Elumalai K, Krishnappa K, Anandan A. Mosquito larvicidal, ovicidal, and repellent properties of botanical extracts against Anopheles stephensi, Aedes aegypti, and Culex quinquefasciatus (Diptera: Culicidae). Parasitology research. 2011 Aug 1;109(2):353-67. https://doi.org/10.1007/s00436-011-2263-1

44. Kumar P, Mishra S, Malik A, Satya S. Insecticidal properties of Mentha species: a review. Industrial Crops and Products. 2011 Jul 1;34(1):802-17. https://doi.org/10.1016/j.indcrop.2011.02.019

45. Kovendan K, Murugan K, Shanthakumar SP, Vincent S, Hwang JS. Larvicidal activity of Morinda citrifolia L. (Noni) (Family: Rubiaceae) leaf extract against Anopheles stephensi, Culex quinquefasciatus, and Aedes aegypti. Parasitology research. 2012 Oct 1;111(4):1481-90. https://doi.org/10.1007/s00436-012-2984-9

46. Kovendan K, Murugan K, Vincent S. Evaluation of larvicidal activity of Acalypha alnifolia Klein ex Willd. (Euphorbiaceae) leaf extract against the malarial vector, Anopheles stephensi, dengue vector, Aedes aegypti and Bancroftian filariasis vector, Culex quinquefasciatus (Diptera: Culicidae). Parasitology research. 2012 Feb 1;110(2):571-81. https://doi.org/10.1007/s00436-011-2525-y

47. Kovendan K, Murugan K, Kumar KP, Panneerselvam C, Kumar PM, Amerasan D, Subramaniam J, Vincent S. Mosquitocidal properties of Calotropis gigantea (Family: Asclepiadaceae) leaf extract and bacterial insecticide, Bacillus thuringiensis, against the mosquito vectors. Parasitology research. 2012 Aug 1;111(2):531-44. https://doi.org/10.1007/s00436-012-2865-2

48. Kumar S, Warikoo R, Mishra M, Seth A, Wahab N. Larvicidal efficacy of the Citrus limetta peel extracts against Indian strains of Anopheles stephensi Liston and Aedes aegypti L. Parasitology research. 2012 Jul 1;111(1):173-8. https://doi.org/10.1007/s00436-011-2814-5

49. Subramaniam J, Kovendan K, Kumar PM, Murugan K, Walton W. Mosquito larvicidal activity of Aloe vera (Family: Liliaceae) leaf extract and Bacillus sphaericus, against Chikungunya vector, Aedes aegypti. Saudi journal of biological sciences. 2012 Oct 1;19(4):503-9. https://doi.org/10.1016/j.sjbs.2012.07.003

50. Tennyson S, Ravindran KJ, Arivoli S. Bioefficacy of botanical insecticides against the dengue and chikungunya vector Aedes aegypti (L.) (Diptera: Culicidae). Asian Pacific Journal of Tropical Biomedicine. 2012 Jan 1;2(3): S1842-4. https://doi.org/10.1016/S22211691(12)60505-X

51. Veni T, Pushpanathan T, Mohanraj J. Larvicidal and ovicidal activity of Terminalia chebula Retz. (Family: Combretaceae) medicinal plant extracts against Anopheles stephensi, Aedes aegypti and Culex quinquefasciatus. Journal of Parasitic Diseases. 2017 Sep 1;41(3):693-702. https://doi.org/10.1007/s12639-016-0869-z
52. Ali SI, Gopalakrishnan B, Venkatesalu V. Evaluation of larvicidal activity of Senecio laetus Edgew. against the malarial vector, Anopheles stephensi, dengue vector, Aedes aegypti and Bancroftian filariasis vector, Culex quinquefasciatus. South African Journal of Botany. $2018 \quad$ Jan 31; 114:117-25. https://doi.org/10.1016/j.sajb.2017.10.018

53. Maradufu A, Lubega R, Dorn F. Isolation of (5E)Ocimerone, a mosquito larvicide from Tagetes minuta. Lloydia. 1978; 41:181-3.

54. Schmutterer H. Some properties of components of the neem tree (Azadirachta indica) and their use in pest control in developing countries [natural pesticide; repellent and phagodeterrent effects; negative effect on the fecundity of some insects; growth-disrupting effect]. Rijksuniversiteit Faculteit Landbouwwetenschappen, Gent. 1981

55. Ameen M, Shahjahan RM, Khan HR, Chowdhury AK. Toxicity of rotenone extracted from indigenous Derris roots on mosquito larvae. J Bangladesh Acad Sci. 1983; 7:39-47.

56. Banerji A, Luthria DL, Kokate SD. Toxicity of capillin, the insecticidal principle of Artemisia nilagirica Clarke. Indian journal of experimental biology. 1990;28(6):588-9.

57. Evans DA, Raj RK. Larvicidal efficacy of Quassin against Culex quinquefasciatus. The Indian journal of medical research. 1991 Sep; 93:324-7.

58. Chauret DC, Bernard CB, Arnason JT, Durst T, Krishnamurty HG, Sanchez-Vindas P, Moreno N, San Roman L, Poveda L. Insecticidal neolignans from Piper decurrens. Journal of Natural Products. 1996 Feb 22;59(2):152-5. https://doi.org/10.1021/np960036y

59. Muthukrishnan J, Seifert K, Hoffmann KH, Lorenz MW. Inhibition of juvenile hormone biosynthesis in Gryllus bimaculatus by Glycosmis pentaphylla leaf compounds. Phytochemistry. 1999 Jan 26;50(2):249-54. https://doi.org/10.1016/S0031-9422(98)00537-8

60. Zebitz CP. Effect of some crude and azadirachtinenriched neem (Azadirachta indica) seed kernel extracts on larvae of Aedes aegypti. Entomologia experimentalis et applicata. 1984 Jan;35(1):11-6. https://doi.org/10.1111/j.1570-7458.1984.tb03351.x

61. Qureshi SA, Mohiuddin SH, Fatima B, Badar Y. Laboratory studies on some plant extracts as mosquito larvicides. Pakistan Journal of Scientific and Industrial Research (Pakistan). 1986 29;361-5.

62. Sccftwartz AM, Paskewitz SM, Orth AP, Tesch MJ, Toong IY, Goodman WG. The lethal effects of Cyperus iria on Aedes aegypti. J Am Mosq Contr Assoc 1998 14;78 - 82.

63. Mwangi RW, Mukiama TK. Evaluation of Melia volkensii extract fractions as mosquito larvicides. Journal of the American Mosquito Control Association. 1988 Dec;4(4):442-7.

64. Saxena SC, Yadav RS. A new plant extract to suppress the population of yellow fever and dengue vector Aedes aegypti L. (Diptera: Culicidae). Current Science. 1983 Aug 5:713-5.

65. Muthukrishnan J, Pushpalatha E. Effects of plant extracts on fecundity and fertility of mosquitoes. Journal of Applied Entomology. 2001 Mar;125(1-2):31-5. https://doi.org/10.1046/j.1439-0418.2001.00503.x 